Proceedings of the 18th National Conference on Superconductivity, Krynica Morska, Poland, October 8-13, 2017

\title{
Tests of Parallel Connected HTS 2G Tapes for Fault Current Limiters
}

\author{
M. MAJKA ${ }^{a, *}$ AND J. KOZAK ${ }^{b}$ \\ ${ }^{a}$ Lublin University of Technology, Institute of Electrical Engineering and Electrotechnologies, \\ Nadbystrzycka 38A, 20-618 Lublin, Poland \\ ${ }^{b}$ Electrotechnical Institute, M. Pozaryskiego 28, 04-703 Warsaw, Poland
}

\begin{abstract}
The superconducting fault current limiters at high voltage levels and for high current applications should be constructed with modular structure. The superconductor tapes have to be connected in series according to voltage condition and in parallel in order to increase the nominal current of the devices. The process of quenching may occur in all superconducting devices, but in fault current limiters this process must be fully controlled. Uncontrolled transition of the superconducting tape from the superconducting state to the resistive state may cause local overheating and burning through of the high-temperature superconducting tape and, consequently, lead to the destruction of the entire superconducting device. The knowledge about these phenomena in order to be able to properly design superconducting fault current limiters is indispensable. In this study, fault currents were applied to the parallel-connected YBCO tapes in a liquid nitrogen bath, and the characteristics of the current distribution were examined experimentally. The tests were carried out for $2 \mathrm{G}$ high-temperature superconducting tapes with copper stabilizer and on tapes without the copper stabilizer.
\end{abstract}

DOI: 10.12693/APhysPolA.135.19

PACS/topics: 84.71.Mn

\section{Introduction}

A rapid increase of the resistance of a superconductor on crossing the current critical value $I_{c}$ makes it possible to build reliable superconducting fault current limiters (SFCLs). SFCLs react very rapidly by limiting the first, the most dangerous, surge current during a current fault condition, thus protecting the devices of the electric power network from the dynamic effects of current faults [1-4]. Superconducting tapes with a copper stabilizer or without $[5,6]$ can be used for the construction of current limiters.

Superconducting tape for SFCLs should be characterised with a high value of the critical current in relation to its width, and with high resistivity in the resistive state. Moreover, superconducting tapes should ideally have identical electrical parameters, i.e. constant value of critical current and resistance in the resistive state, on its entire length. In order to make the superconducting elements of SFCLs, superconducting tapes are connected in series and in parallel. Connecting superconducting tapes in parallel is used in order to multiply the critical current of the superconducting element. The main objective of the tests was to experimentally determine quench differences for tapes with and without a copper stabilizer and for the distribution of current in HTS tapes connected in parallel.

*corresponding author; e-mail: m.majka@pollub.pl

\section{Characteristics of HTS tapes used for tests}

Two kinds of $2 \mathrm{G}$ HTS tapes have been used for the investigation of the transient response of the $2 \mathrm{G}$ hightemperature superconducting (HTS) tape under an alternating overcurrent. Series of measurements were carried out for tapes with a copper stabilizer (SCS SuperPower tape) and on tapes without a copper stabilizer (SF SuperPower tape). Tape parameters are collected in Table I.

TABLE I

Specification of used 2G HTS tapes

\begin{tabular}{l|c|c}
\hline \hline Type of 2G HTS wire & SF4050 & SCS4050 \\
\hline producer & SuperPower & SuperPower \\
width & $4 \mathrm{~mm}$ & $4 \mathrm{~mm}$ \\
substrate thickness & $0.050 \mathrm{~mm}$ & $0.050 \mathrm{~mm}$ \\
HTS thickness & $1 \mu \mathrm{m}$ & $1 \mu \mathrm{m}$ \\
stabilizer thickness & $2 \mu \mathrm{m}$ & $2 \mu \mathrm{m}$ \\
cap layer & silver & silver \\
copper stabilization & without & $40 \mu \mathrm{m}$ \\
average $I_{c}$ & $120 \mathrm{~A}$ & $126 \mathrm{~A}$ \\
minimum $I_{c}$ & $118 \mathrm{~A}$ & $123 \mathrm{~A}$ \\
maximum $I_{c}$ & $122 \mathrm{~A}$ & $129 \mathrm{~A}$ \\
substrate resistivity & $125 \mu \Omega \mathrm{cm}$ & $125 \mu \Omega \mathrm{cm}$
\end{tabular}

The $4 \mathrm{~mm}$ width tapes were used for measurements because our measuring system has a limited short-circuit current. The maximum short-circuit current in the laboratory circuit was $600 \mathrm{~A}$. Experimentally determined temperature-dependent resistance between $77 \mathrm{~K}$ and a room temperature for these tapes is presented in Fig. 1. The measurement of the superconducting tape resistance was performed using the 4 -wire method. 

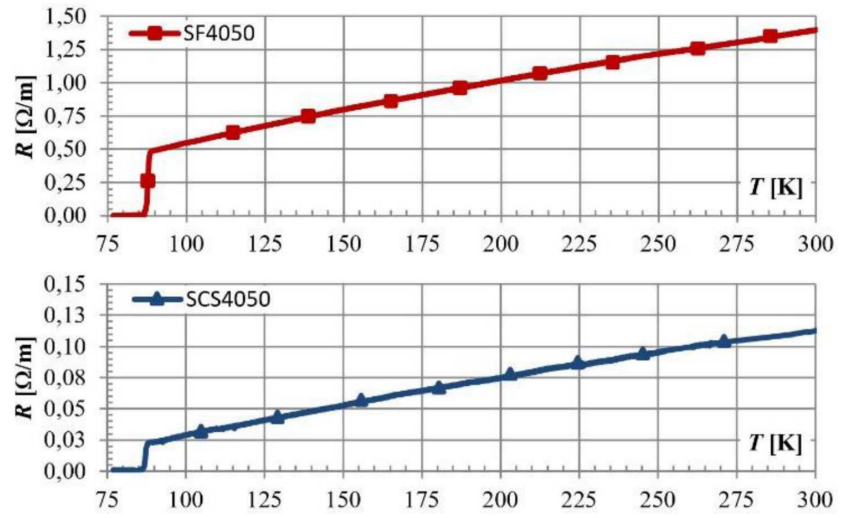

Fig. 1. Measured temperature-dependent resistance of $4 \mathrm{~mm}$ wide SCS4050 and SF4050 tapes (length of tape: $100 \mathrm{~cm})$.

\section{Test circuit}

The experimental setup consisted of three main elements: a holder with the examined tapes, a supplying system, and a data acquisition system. A schematic diagram of the experimental setup is presented in Fig. 2. The measuring system allowed to change the voltage powering of the superconducting tape and to start and stop the power supply automatically. It is possible to adjust the voltage in the short-circuit testing station thanks to using an autotransformer. The controller has a system of detecting the passage of the signal through zero value, a system of regulating the time of delay and a system of regulating the time of a short-circuit. The powering and recording systems will be controlled via the data acquisition system equipped with PC computer, a National Instrument DAQ Card and software written in LabView environment [7].

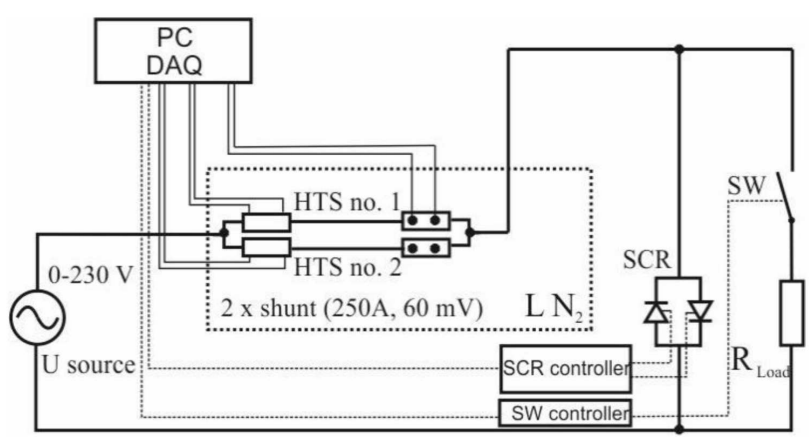

Fig. 2. Schematic diagram of $\mathrm{AC}$ over-current test setup.

The holder for testing of the HTS tapes in transient state is presented in Fig. 3. Copper terminals were soldered to a $16.5 \mathrm{~cm}$ long piece of a superconducting tape. The electrical termination of the parallel connected tape samples with copper stabilizer and without stabilizer has been identical. The copper plates have been soldered on both sides of the HTS tape using Sn62Pb36Ag2 alloy.

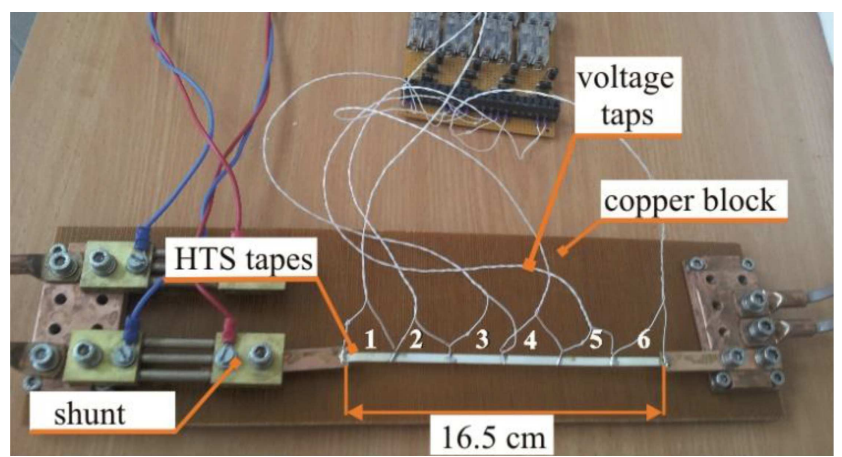

Fig. 3. The holder for testing of the HTS tape in transient state: $16.5 \mathrm{~cm}$ long tape with voltage leads soldered to the tape at $2.75 \mathrm{~cm}$.

$250 \mathrm{~A} 60 \mathrm{mV}$ shunt was used to test the current in the circuit. In order to evaluate the longitudinal quench propagation seven voltage taps have been soldered to the HTS tape to monitor the voltage drop along the tape. The voltages were measured differentially on each segment of the tape. The segments of the tape and voltages of these segments were numbered as shown in Fig. 3. A measuring length of the superconducting tape was mounted in the holder and then cooled with liquid nitrogen in a cryostat under atmospheric pressure. Tests of the superconducting tape have been performed for several values of the current below its critical value $I_{c}$ and for currents whose values exceed several times the tape's critical current value.

A holder used for the tests of two superconducting tapes connected in parallel is presented in Fig. 4. It consists of a base to which two copper blocks were screwed.

Two $16.5 \mathrm{~cm}$ (distance between current connectors) pieces of HTS tape were used in the tests. Copper terminals were soldered to the ends of the HTS tapes and screwed to shunts from one end and to copper block from the other end. Two voltage taps, with distance of $10 \mathrm{~cm}$ between them, were soldered to the HTS tape. In order to monitor the current flowing through each tape, two $250 \mathrm{~A}, 60 \mathrm{mV}$ shunts were used. Before tests both shunts were cooled down to $77 \mathrm{~K}$ and calibrated for better measurement accuracy. The resistance of the shunts

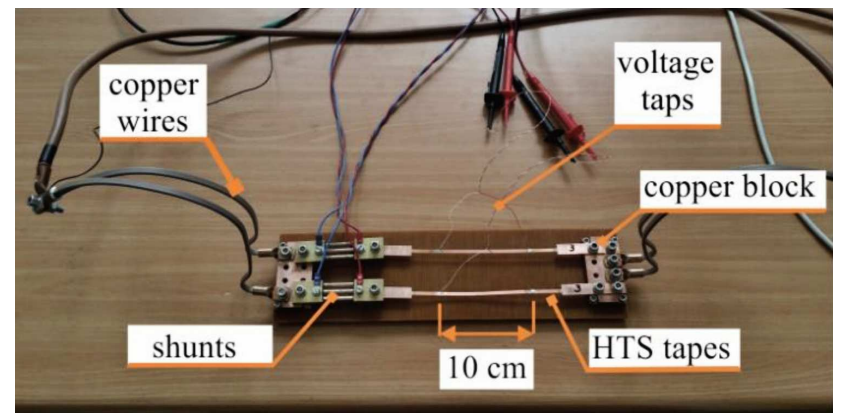

Fig. 4. The holder for testing of HTS tapes connected in parallel. 
are $222 \mu \Omega$ and $223 \mu \Omega$ at $77 \mathrm{~K}$. The same soldering method was used when preparing HTS tape samples, as well as copper wires of the same length and the same screw torque. During tests, the holder was cooled in a liquid nitrogen bath. Tests were performed for different values of the powering voltage and fault currents.

\section{Results and discussion}

\subsection{The quench propagation in single HTS tape}

Figure 5 presents the measurements results for SCS4050 tape with a copper stabilizer. For increasing values of current (Fig. $5 \mathrm{a}-\mathrm{C}$ ) at particular sections of the superconducting tape, identical current waveforms were recorded, thus the tape uniformly goes from the resistive state and uniformly heats up on its entire length. The root mean square (RMS) values of current were determined for all current waveforms in Figs. 5-8. If the RMS value of current is higher, the superconductor tape heats up to a higher temperature at the same time.

Figures $6 \mathrm{a}$ and $6 \mathrm{~b}$ indicates an early quench of sections 3, 4, 5 and 6 . Therefore, the quench for this tape is not uniform. Particularly dangerous is the passage of

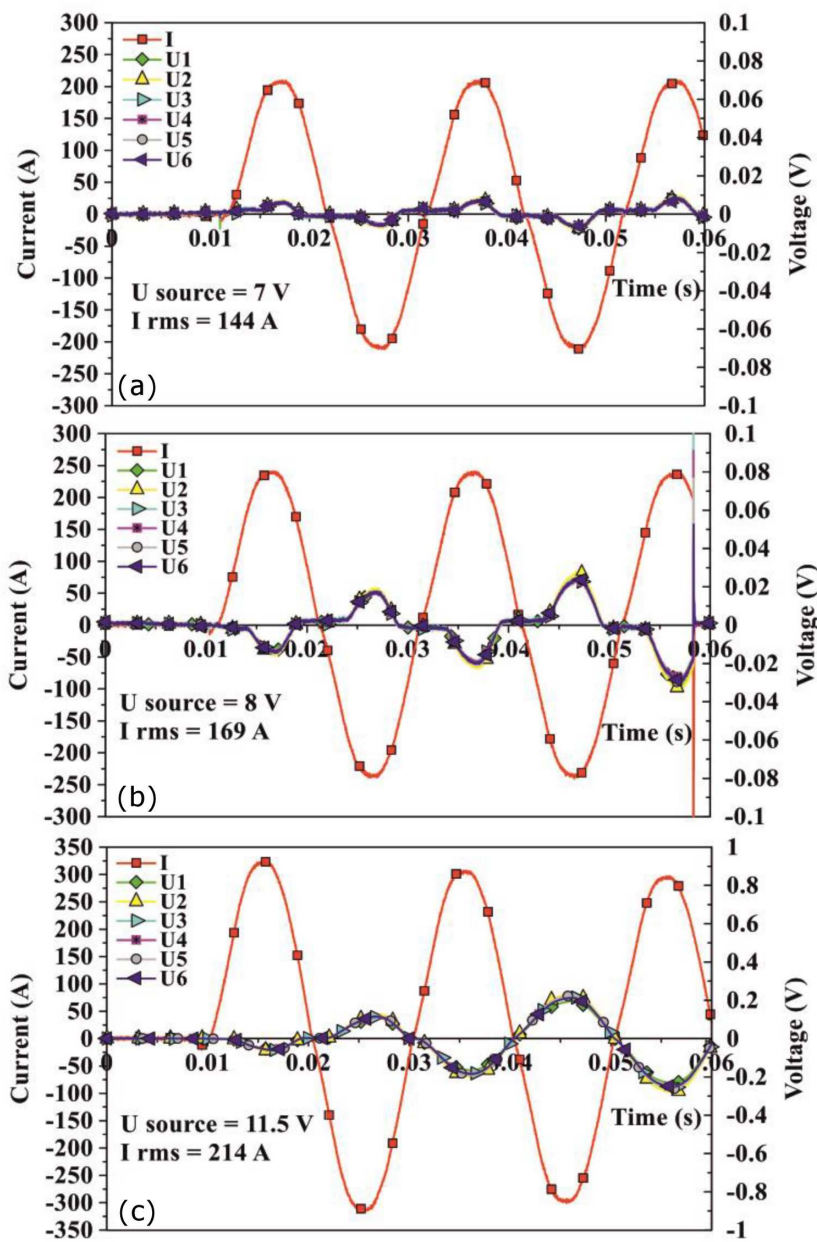

Fig. 5. Current waveform and voltages at subsequent sections of SCS4050 tape with a copper stabilizer.

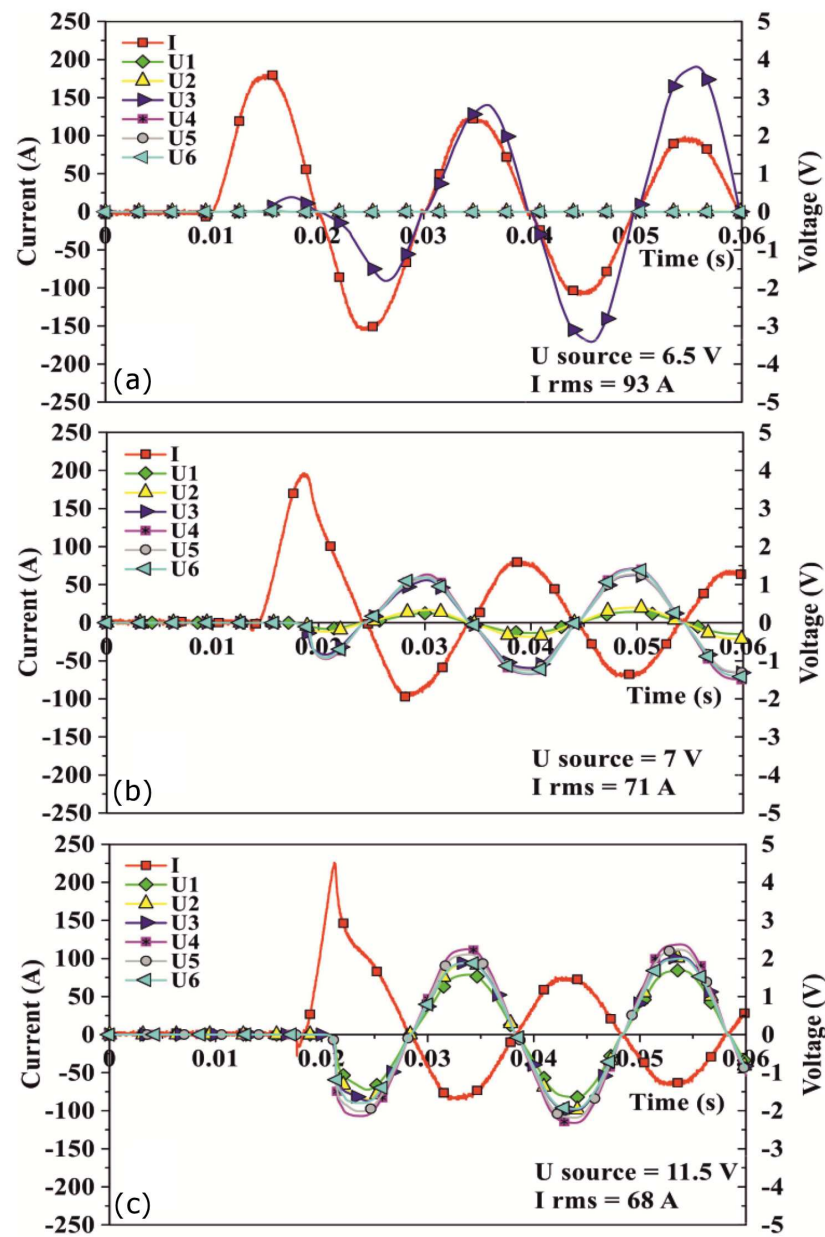

Fig. 6. Current waveforms and measured voltages at subsequent sections of SF4050 tape without a copper stabilizer.

a current below $200 \mathrm{~A}$ and the occurrence of quench in just one segment of the tape (Fig. 6a). Segment 3 presents a higher voltage during the fault period in Fig. 6a, probably this segment has the lowest critical current value than the other segments of the sample. In this case, the superconducting tape heats up drastically over a small section and may undergo thermal destruction. For higher current values, quench is more uniform, and from a particular voltage level $\left(U_{n o m} / l=46 \mathrm{~V}_{r m s} \mathrm{~m}^{-1}\right.$ for SF4050 and $2 \mathrm{~V}_{r m s} \mathrm{~m}^{-1}$ for SCS4050) all sections of the superconducting tape move into the resistive state and heat up almost uniformly (Fig. 6c). The quench is more homogeneous in the tape with copper stabilization due to bypassing the current through the copper stabilizer during the quench $[6,7]$.

\subsection{Tests of HTS 2G tapes connected in parallel}

Distribution of currents in connected in parallel SCS4050 tapes with a copper stabilizer is uniform (Fig. 7) but tape no. 2 goes into the resistive state sooner than tape no. 1, and the voltage at it is by $30 \%$ higher than at tape no. 1 (Fig. 7c). 

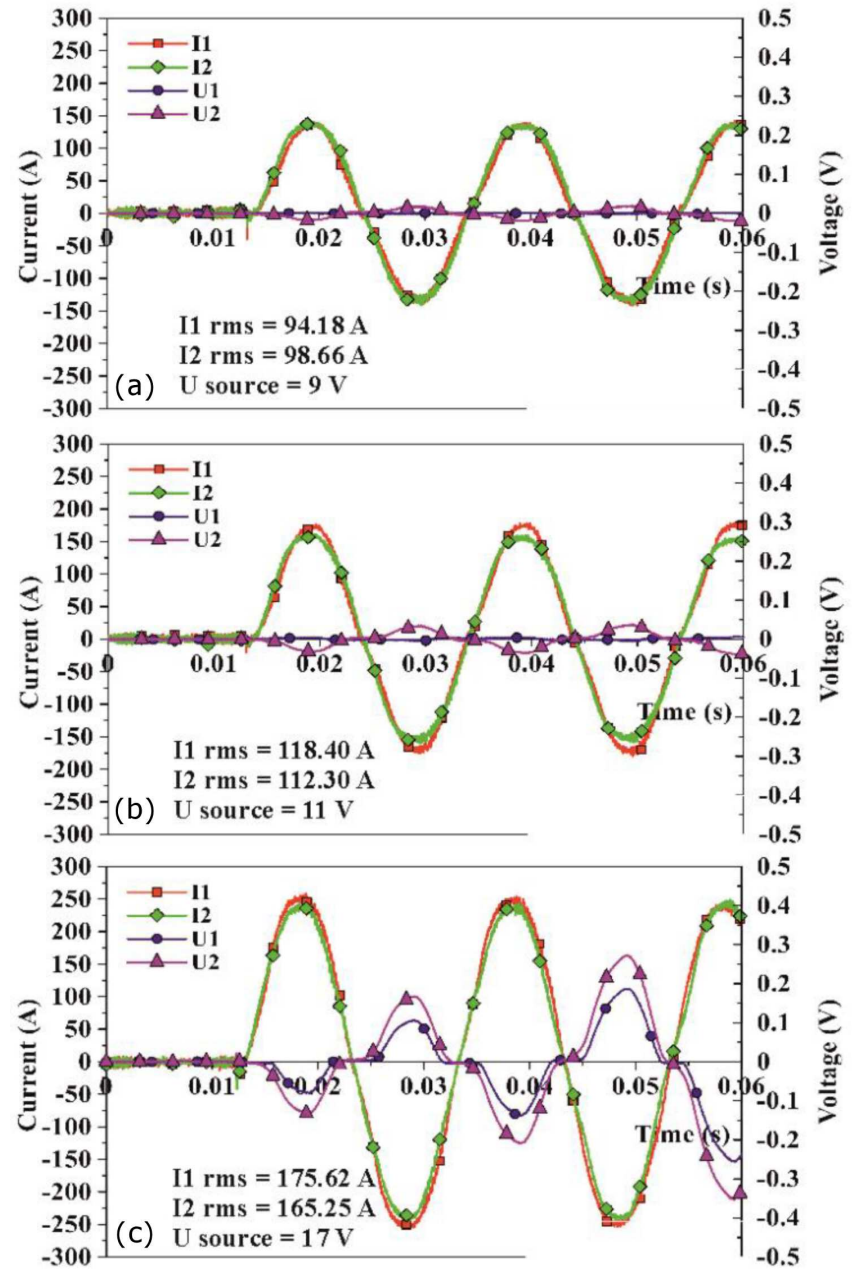

Fig. 7. Waveforms of voltages and currents for two connected in parallel SCS4050 tapes with copper stabilizers.

In case of two connected in parallel SF4050 tapes without a copper stabilizer, current distribution between the tapes was uneven for all the tested samples (Fig. 8). If the current passing through tape no. 2 is not much higher than the critical current (Fig. 8a), then the current passing through tape no. 1 is almost 3 times lower than that passing through tape no. 2. Uneven passage of currents between the tapes could be caused by the differences in resistance of twisted connections or the soldered connections between the superconducting tapes. For higher values of current (Fig. 8b), HTS tape no. 2 goes into the resistive state sooner than tape no. 1. For currents twice higher than the critical current, the redistribution of currents is balanced out (Fig. 8c) and the recorded voltage at tape no. 2 is several times higher than at tape no. 1. Uneven current distribution in two parallel connected identical HTS tapes with the same critical current is caused by the differences in resistance of solder joints between the copper terminal and HTS tape.

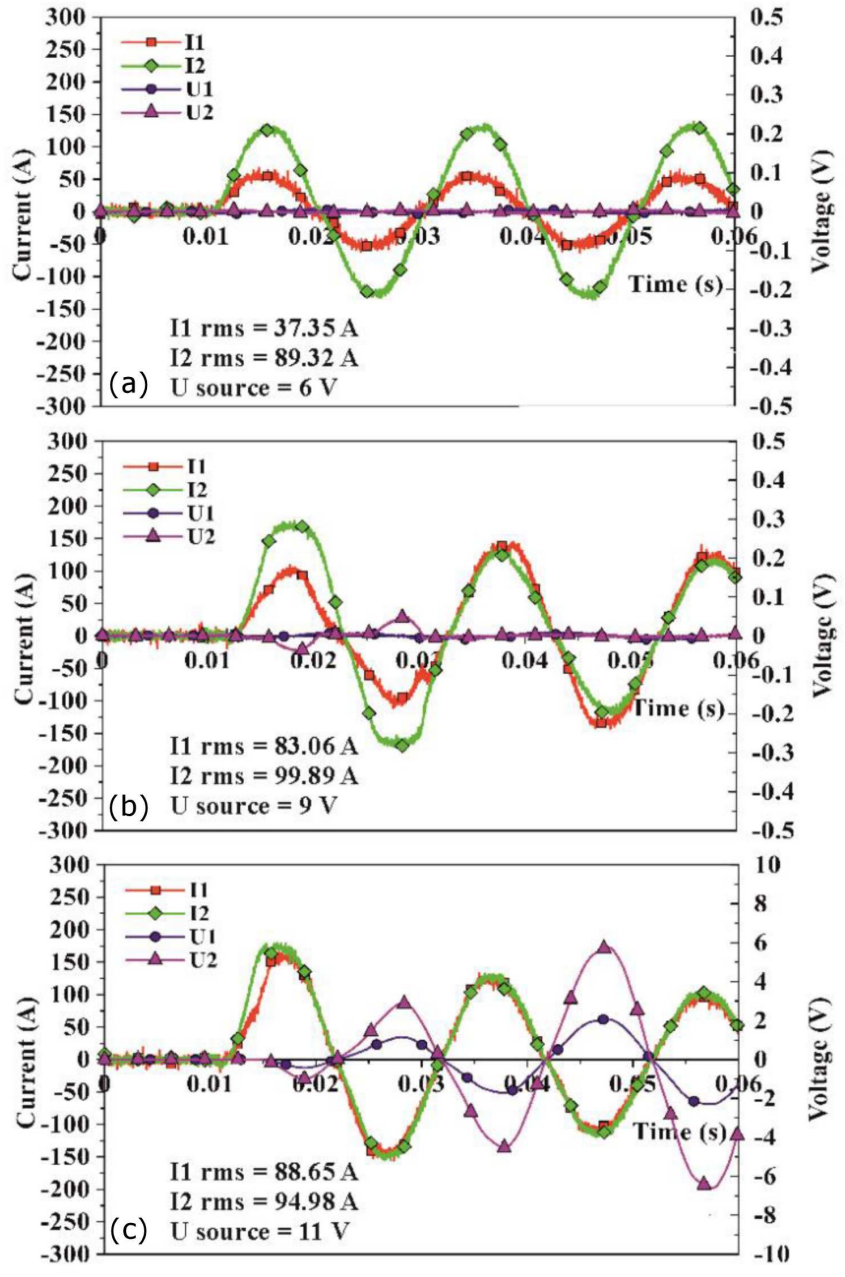

Fig. 8. Waveforms of voltages and currents for two connected in parallel SF4050 tapes without copper stabilizers.

\section{Conclusion}

The aim of this article was to find the transient response of the $2 \mathrm{G}$ high-temperature superconducting tape and current distribution in parallel connected tapes. The series of measurements were carried out for $2 \mathrm{G}$ HTS tapes with a copper stabilizer and on tapes without a copper stabilizer. Differences in the distribution of currents resulting from contact of connector resistance were observed for superconducting tapes connected in parallel. Not only quench for a single SCS 4050 tape but also currents distribution for HTS tapes with a copper stabilizer connected in parallel are more uniform than for tapes without a copper stabilizer. The HTS tapes without copper stabilizer (e.g. SuperPower SF type) more effectively limits the short circuit current and heats up to a lower temperature than the HTS tape with copper stabilizer, due to the higher resistance value after quench. 


\section{References}

[1] A. Morandi, Physica C 484, 242 (2013).

[2] J. Kozak, M. Majka, S. Kozak, T. Janowski, IEEE Trans. Appl. Supercond. 23, 5600604 (2013).

[3] M. Majka, J. Kozak, S. Kozak, G. Wojtasiewicz, T. Janowski, IEEE Trans. Appl. Supercond. 25, 5601005 (2015).

[4] O. Naeckel, M. Noe, IEEE Trans. Appl. Supercond. 24, 5601605 (2014).
[5] M. Majka, S. Kozak, Przegl. Elektrotechn. 85, 183 (2009).

[6] M. Majka, J. Kozak, S. Kozak, IEEE Trans. Appl. Supercond. 27, 5601405 (2017).

[7] D. Czerwinski, L. Jaroszynski, T. Janowski, M. Majka, J. Kozak, IEEE Trans. Appl. Supercond. 24, 5600104 (2014). 